\title{
Antimicrobial resistance patterns of bovine Salmonella enterica isolates submitted to the Wisconsin Veterinary Diagnostic Laboratory: 2006-2015
}

\author{
J. R. Valenzuela, ${ }^{*}$ A. K. Sethi, $\dagger$ N. A. Aulik, $\ddagger$ and K. P. Poulsen $\S^{1}$ \\ *Wisconsin Veterinary Diagnostic Laboratory, Madison 53706 \\ †Department of Population Health Sciences, University of Wisconsin-Madison, Madison 53726 \\ ‡Department of Pathobiological Sciences, and \\ $\S M e d i c a l$ Sciences Department, University of Wisconsin-Madison, Madison 53706
}

\begin{abstract}
Salmonellosis on the dairy continues to have a significant effect on animal health and productivity and in the United States. Additionally, Salmonella enterica ssp. enterica causes an estimated 1.2 million cases of human illness annually. Contributing to the morbidity and mortality in both human and domestic animal species is emergence of antimicrobial resistance by Salmonella species and increased incidence of multidrug-resistant isolates. This study describes serotype distribution and the antimicrobial resistance patterns for various Salmonella serotypes isolated from bovine samples submitted to the Wisconsin Veterinary Diagnostic Laboratory (WVDL) over the past $10 \mathrm{yr}$. Salmonella serotyping and antimicrobial susceptibility testing data were obtained from the laboratory information management system at WVDL. Data from accessions were limited to bovine samples submitted to the WVDL between January 2006 and June 2015 and those that had both a definitive serotype and complete results for antimicrobial susceptibility testing. A total of 4,976 isolates were identified. Salmonella enterica ser. Dublin was the most prevalent serotype identified among bovine samples submitted to the WVDL, accounting for a total of 1,153 isolates (23\% of total isolates) over the study period. Along with Dublin, Salmonella enterica ser. Cerro (795, 16\%), Newport (720, 14\%), Montevideo (421, 8\%), Kentucky (419, $8 \%)$, and Typhimurium (202, 4\%) comprised the top 6 most commonly isolated serotypes during that time. Overall, resistance of bovine Salmonella isolates in the study population remained stable, although decreases in resistance were noted for gentamicin, neomycin, and trimethoprim sulfamethoxazole during the study
\end{abstract}

Received May 8, 2016.

Accepted October 30, 2016.

${ }^{1}$ Corresponding author: keith.poulsen@wvdl.wisc.edu period. All isolates remained susceptible to enrofloxacin. These data show that antimicrobial susceptibility for bovine Salmonella has changed in the population served by WVDL in the past $10 \mathrm{yr}$. This information is important for understanding Salmonella disease ecology in Wisconsin. Our findings are also relevant for animal and public health by improving informed antimicrobial use, new drug development, and regulation of their use in food animals.

Key words: bovine, Salmonella, antimicrobial resistance, dairy, Wisconsin

\section{INTRODUCTION}

Salmonella causes significant disease in food animals and those animals are also potential sources of zoonotic human infection (Mandal and Brennand, 1988). Common clinical manifestations of Salmonella infection in cattle include diarrhea, pneumonia, abortion, and death. Infection in dairy cattle is also associated with decreased milk production (Nielsen et al., 2012, 2013; O'Doherty et al., 2015). Salmonellosis can have a substantial economic effect on dairies in the United States, with some financial loss estimates ranging in the billions of dollars annually (Peters, 1985; Huston et al., 2002). Although cattle can be infected by several Salmonella enterica serotypes, the majority of infections are attributed to serovar Dublin, which tends to cause more systemic infections, and serovar Typhimurium, more often causing enteritis in young calves (Costa et al., 2012).

Salmonella enterica is also of significant public health interest with zoonotic potential. The majority of infections in humans are associated with products derived from food animals and contaminated produce, which has been linked to domestic and feral animal populations (Jay-Russell et al., 2014; Haack et al., 2016). Most cases of Salmonella in humans are likely to be self-limiting; however, severe illness has been seen in children, the elderly, and immune-compromised patients, which 
require antibiotic treatment (CDC, 2013b). Annually, the United States has approximately 100,000 infections with a resistant strain of non-typhoidal Salmonella (CDC, 2013b). Human salmonellosis costs the US economy approximately $\$ 2$ to $\$ 4$ billion through loss of work, health care costs, and morbidity (Foley and Lynne, 2007).

Effective antimicrobial stewardship is contingent, in part, on ongoing surveillance of antimicrobial resistance trends in food products, animals, and people. This information can better inform public policy as well as clinical practice regarding appropriate antimicrobial use. Trends in antimicrobial resistance of Salmonella isolates from food animals are of public health concern given the potential for the spread of resistant microorganisms to people. Thus, the objective of our study was to describe the epidemiology and antimicrobial resistance patterns for bovine Salmonella enterica serotypes isolated from samples submitted to the Wisconsin Veterinary Diagnostic Laboratory (WVDL) over the last $10 \mathrm{yr}$.

\section{MATERIALS AND METHODS}

\section{Data Collection}

Data were retrospectively collected on all bovine Salmonella enterica isolates identified at the WVDL between January 1, 2006, and July 30, 2015, using the Laboratory Information Management System (UVIS, Ross Group Inc., Dayton, OH). Antimicrobial susceptibility testing (AST) data were queried from antimicrobial resistance databases at the WVDL Madison and Barron locations (Sensititer, Thermo Fisher Scientific, Waltham, MA). The WVDL is 1 of 3 laboratories that provide testing and diagnostic services to veterinarians and producers in the state. Approximately $90 \%$ of the total caseload is from the state of Wisconsin, which is dominated by the dairy industry. Salmonella enterica is typically associated with such clinical presentations as calf and adult cow diarrhea, abortion workups, and septicemia in both adult cows and calves. The organism is also often associated with nonspecific herd health issues or endemic disease problems, in particular involving Salmonella enterica ser. Dublin. Sample types included feces submitted for routine diagnostics and tissues harvested during necropsy. Milk was excluded from our study samples because it is unknown how often Salmonella enterica is shed in milk, but it is thought to be rare and attributed mostly to postcollection contamination (Nielsen, 2013; Van Kessel et al., 2013). Diagnostic specimens are required to be submitted by veterinarians for interpretation of results for the purposes of disease management.
Due to the financial constraints of submitting clients, WVDL only fully serotypes and provides sensitivity for 1 Salmonella serotype isolated from a single accession, which can include multiple animals or tissues. All Salmonella isolates are serogrouped to confirm that all Salmonella isolated from a single accession or animal are of the same serogroup, and then one of the Salmonella isolated is fully serotyped. These accessions are considered single accessions, as just 1 serogroup of Salmonella was identified. If an accession or animal had more than 1 serogroup, each serogroup would have 1 isolate serotyped and susceptibility assessed. These accessions are considered multiple accessions, as more than 1 Salmonella serotype was identified. Each serotype would be given a unique identification number composed of the original accession number followed by an animal and specimen number. In rare cases, the same accession or animal might have had more than 1 isolate fully serotyped and susceptibility performed even if the serotype was the same. For these accessions, only those isolates with unique AST results were included.

All serotyping was performed from isolated colonies following the Kauffman-White classification system. Identification of Salmonella Dublin using real-time reverse transcription PCR (RT-PCR) was introduced at the laboratory in the summer of 2014. Dublin isolates with high $(>35)$ cycle threshold $\left(\mathbf{C}_{\mathbf{T}}\right)$-positive RT-PCR values were not included in this data set because they are generally not able to be successfully cultured to be subject to AST. Low $(<35) \mathrm{C}_{\mathrm{T}}$-positive RT-PCR samples were automatically cultured, serotyped, subject to AST, and included in this study. The internal success rate is estimated to be 70 to $75 \%$ for successful post-PCR culture of samples with $\mathrm{C}_{\mathrm{T}}$ lower than 35 for both the Salmonella species and Salmonella Dublin multiplex PCR.

Isolates that were identified as Salmonella Bardo were counted as Salmonella Newport based on recent recommendations by the Centers for Disease Control and Prevention on interpretation of Salmonella serotypes, which describes this pair as genetically indistinguishable (CDC, 2013a). The year of the finalized date was used for analysis for accessions spanning change of the calendar year.

\section{Antimicrobial Susceptibility}

Isolates were included if they were fully serotyped, using standard operating procedures reviewed and accredited by the American Association of Veterinary Laboratory Diagnosticians, and subject to AST performed using the bovine or porcine MIC format plate with tulathromycin [Sensititer BOPO6F (post-2007) or BOPO1F (pre-2007), Thermo Fisher Scientific, Oak- 
wood Village, $\mathrm{OH}]$, which includes 18 antimicrobial agents (ceftiofur, tiamulin, chlortetracycline, gentamicin, florfenicol, oxytetracycline, penicillin, ampicillin, danofloxacin, sulphadimethoxine, neomycin, trimethoprim/sulfamethoxazole, spectinomycin, tylosin tartrate, tulathromycin, tilmicosin, clindamycin, and enrofloxacin). Notable differences between the BO$\mathrm{PO} 1 \mathrm{~F}$ and the BOPO6F MIC format plate adopted in 2007 by both laboratories for AST included the addition of tulathromycin (7 concentrations), elimination of erythromycin, sulfathiazole, and sulfachloropyridazine, and the modification of the range of concentrations used for tylosin tartrate, gentamicin, trimethoprim sulfamethoxazole, and sulfadimethoxine. Data for tulathromycin was limited to those samples received and reported after 2007. Incompletely serotyped isolates or those for which AST results were not complete or unavailable were excluded.

Antimicrobial susceptibility results included a numerical value corresponding to the MIC and a categorical interpretive criteria of resistant, susceptible, or intermediate based on Clinical and Laboratory Standards Institute (CLSI) standards (CLSI, 2013, 2015). The CLSI standards are updated approximately every 3 to $5 \mathrm{yr}$; the most recent veterinary standards and accompanying tables were published in 2013 and 2015, respectively. Intermediate and resistant categories were combined for analysis. The categorical interpretive criteria (resistant/susceptible/intermediate) are based on breakpoints of the numerical MIC values, which are developed for a specific host-pathogen-drug combination based on available research. No standards are specific to bovine Salmonella enterica for any of the antibiotics tested for on the BOPO1F and BOPO6F MIC format plates, so MIC breakpoints and associated interpretive criteria are often extrapolated from human data, where available. In the event human data are not available, as in the case of tulathromycin, ceftiofur, tylosin tartrate, and danofloxacin, interpretive criteria were not available. For those antibiotics, numerical MIC values may be reported, but results were classified as not interpretable. Given the widespread use of macrolides (e.g., tulathromycin) and cephalosporins (e.g., ceftiofur) in dairy practice, we included tulathromycin and ceftiofur in our analysis, despite the lack of CLSI-established interpretive criteria for these agents. Analysis for these antimicrobial agents was limited to their numerical MIC value.

Minimum inhibitory concentration values for ceftiofur were dichotomized at a value $\geq 8 \mu \mathrm{g} / \mathrm{mL}$, based on the breakpoints for Salmonella enterica listed in the National Antimicrobial Resistance Monitoring System (NARMS) 2011 Annual Animal Report (USDA, 2014). No equivalent breakpoints were found for tulath- romycin and Salmonella enterica specifically; therefore, MIC values for tulathromycin were dichotomized at an MIC value $\geq 32 \mu \mathrm{g} / \mathrm{mL}$, based on the breakpoint for azithromycin in Salmonella isolates published by NARMS (USDA, 2014).

\section{Statistical Analysis}

Data were analyzed using Stata 14.0 (StataCorp, College Station, TX). Yearly numbers of bovine Salmonella as well as serotype-specific frequencies were calculated. Yearly numbers and proportions of resistant isolates by antibiotic agent were tabulated. Resistance data for those antimicrobial agents with CLSI established breakpoints were also tested for significant trends using negative binomial regression analysis with robust standard errors. Year of analysis was the independent variable whereas resistance to that antibiotic was the dependent variable. Statistical analysis of the overall change for each antibiotic is described by an incidence rate ratio and associated $P$-value. A $P$-value of $<0.05$ was considered statistically significant.

The MIC distribution tables were created for antimicrobial agents with interpretive criteria. Prevalence ratios and $P$-values were obtained using negative binomial regression models with robust standard errors to examine per year change in prevalence of resistance for each antimicrobial (Supplemental Tables S1-S9; https://doi.org/10.3168/jds.2016-11419). In the case of ceftiofur and tulathromycin, the overall change in the MIC distributions throughout the study period was analyzed for significance using a Kruskal-Wallis test.

\section{RESULTS}

A total of 4,976 bovine Salmonella enterica isolates with susceptibility results were examined from samples submitted to the WVDL from January 1, 2006, to July 30, 2015 (Table 1). Singles accessions made up $89 \%$ $(4,448)$ and multiple accessions made up $11 \%$ (528) of all isolates analyzed. Ninety-four percent $(4,678)$ of the isolates came from the Madison laboratory and $6 \%$ (289) were isolated at the Barron laboratory. Ninetythree percent $(4,609)$ of all isolates were from the state of Wisconsin. Breed information was available for $94 \%$ $(4,669)$ of the isolates, and $99 \%(4,618)$ of these were identified as dairy breeds. Eighty-six percent $(4,276)$ of all isolates had age-related information, and among these 2,504 (59\%) isolates came from calves and 1,772 (41\%) from adult cattle. Among isolates for which a specific numerical age was recorded $(3,725) ; 38(1 \%)$ were a fetus, $1,983(53 \%)$ were 0 to 8 wk old, $410(11 \%)$ were 9 wk to 12 mo old, 26 (1\%) were between 12 and 20 mo old, and 1,268 (34\%) were over 20 mo of age. 
Table 1. Number (\% in parentheses) of bovine Salmonella enterica isolates, by serotype, tested for antimicrobial susceptibility by the Wisconsin Veterinary Diagnostic Laboratory, 2006-2015

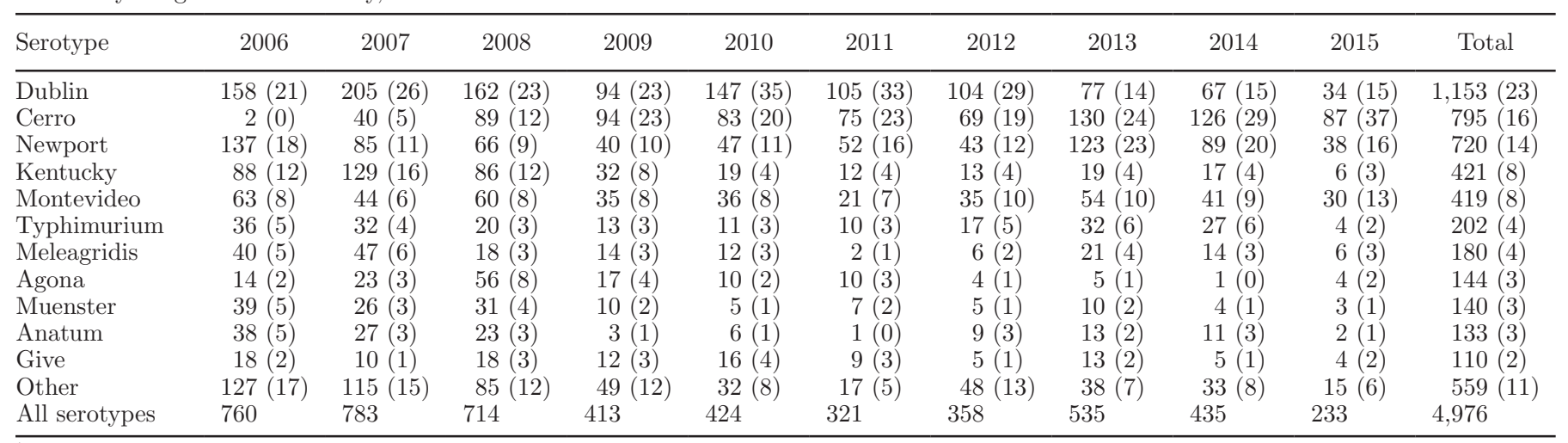

${ }^{1}$ Samples included feces submitted for routine diagnostics and tissues harvested for necropsy. Isolates were included if they were both fully serotyped and had complete antimicrobial susceptibility results.

Table 1 shows the distribution of the most commonly isolated Salmonella enterica serotypes as a number and percent of total isolates that were fully serotyped and tested for antimicrobial susceptibility. Over the entire study period the top 6 most commonly isolated serotypes included Salmonella Dublin, Cerro, Newport, Kentucky, Montevideo, and Typhimurium. Each year the 5 most prevalent serotypes changed slightly. Serotype Dublin was the most common each year until 2012 , ranging from 21 to $35 \%$ of yearly bovine Salmonella enterica isolates. Since 2013 Salmonella Cerro has become the most commonly isolated serotype, followed closely by Salmonella Newport.

The proportion of resistant isolates as counts and as percentages of total yearly Salmonella enterica isolates were divided up by each antimicrobial tested and CLSI drug class, as shown in Table 2. Among all bovine Salmonella enterica isolates detected during the study period, we found a significant decrease in resistance seen in aminoglycosides and trimethoprim sulfamethoxazole (TMS). Tetracyclines, phenicols, and sulfonamides had more modest decreases in resistance. Ampicillin was the only $\beta$-lactam antibiotic with a significant decrease in resistance prevalence. For all other antibiotics evaluated, no significant change in susceptibility was identified over the study period. Of these antibiotics, bovine Salmonella enterica isolates remained nearly $100 \%$ resistant to spectinomycin, penicillin, clindamycin, tilmicosin, and tiamulin. Isolates remained $100 \%$ susceptible to enrofloxacin throughout the study period.

Tables 3, 4, and 5 detail changes in prevalence of Salmonella enterica isolates with resistance to different antibiotics over the study period for the top 3 most prevalent serotypes, Dublin, Cerro, and Newport, respectively. Isolates of Salmonella Cerro showed overall lower levels of resistance to all antibiotics except those for which all isolates maintained nearly $100 \%$ resistance (spectinomycin, penicillin, clindamycin, tilmicosin, and tiamulin). Isolates of Salmonella Newport and Cerro showed similar patterns of high susceptibility to gentamicin, enrofloxacin, and TMS, whereas Dublin showed a relatively higher level of resistance to TMS.

The distribution of yearly counts and percentages of all bovine Salmonella enterica isolates tested against ceftiofur, by numerical MIC category, is outlined in Table 6. A Kruskal-Wallis test performed on this data showed that overall, changes over the study period were statistically significant $\left[\chi^{2}(9)=42.084, P=0.0001\right]$. Table 7 shows the same analysis for tulathromycin MIC values; changes for tulathromycin were also significant $\left[\chi^{2}(8)=552.879, P=0.0001\right]$.

Analysis of changes in resistance for isolates tested against ceftiofur, based on the distribution of MIC values dichotomized at an MIC $\geq 8 \mu \mathrm{g} / \mathrm{mL}$, showed that proportions of resistant isolates decreased starting in 2012 (Figure 1). A similar evaluation of MIC values for tulathromycin, dichotomized at an MIC $\geq 32 \mu \mathrm{g} / \mathrm{mL}$, does not show a clear trend over time (Figure 2), but overall levels of resistance remained low throughout the study period.

Supplemental Tables S1 to S9 (https://doi. org/10.3168/jds.2016-11419) display MIC distribution and results of analysis of per year change in prevalence of resistance for antimicrobial agents with interpretive criteria. Results for changes over the entire study period parallel results seen in analysis of change in percent resistance over that time period. Analysis of trends from 2006 to 2010 compared with 2011 to 2015 show more pronounced changes occurring in latter half of the study period. The number of bovine Salmonella enterica isolates evaluated at the veterinary diagnostic laboratory each year decreased overall, from 783 total 

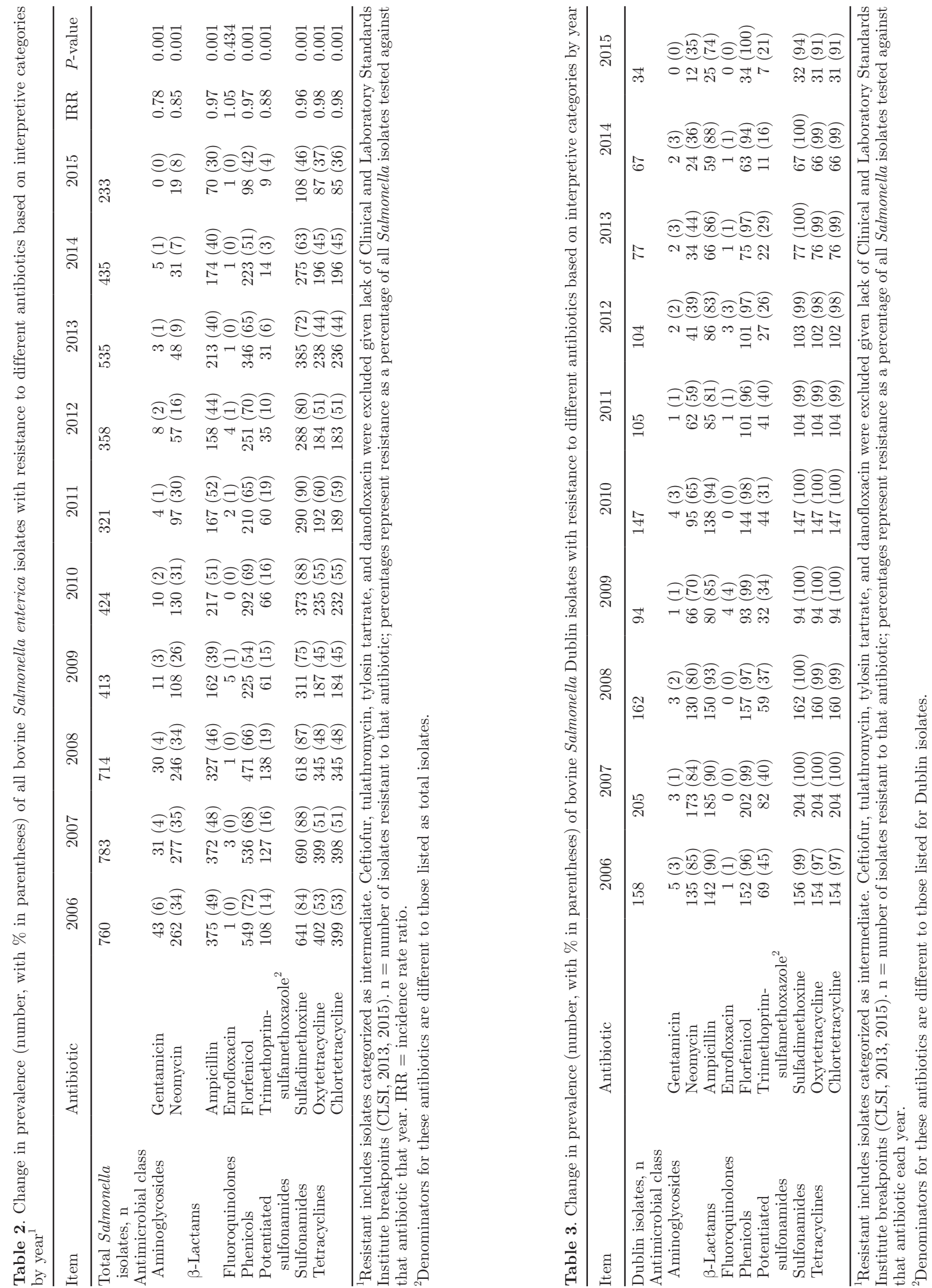

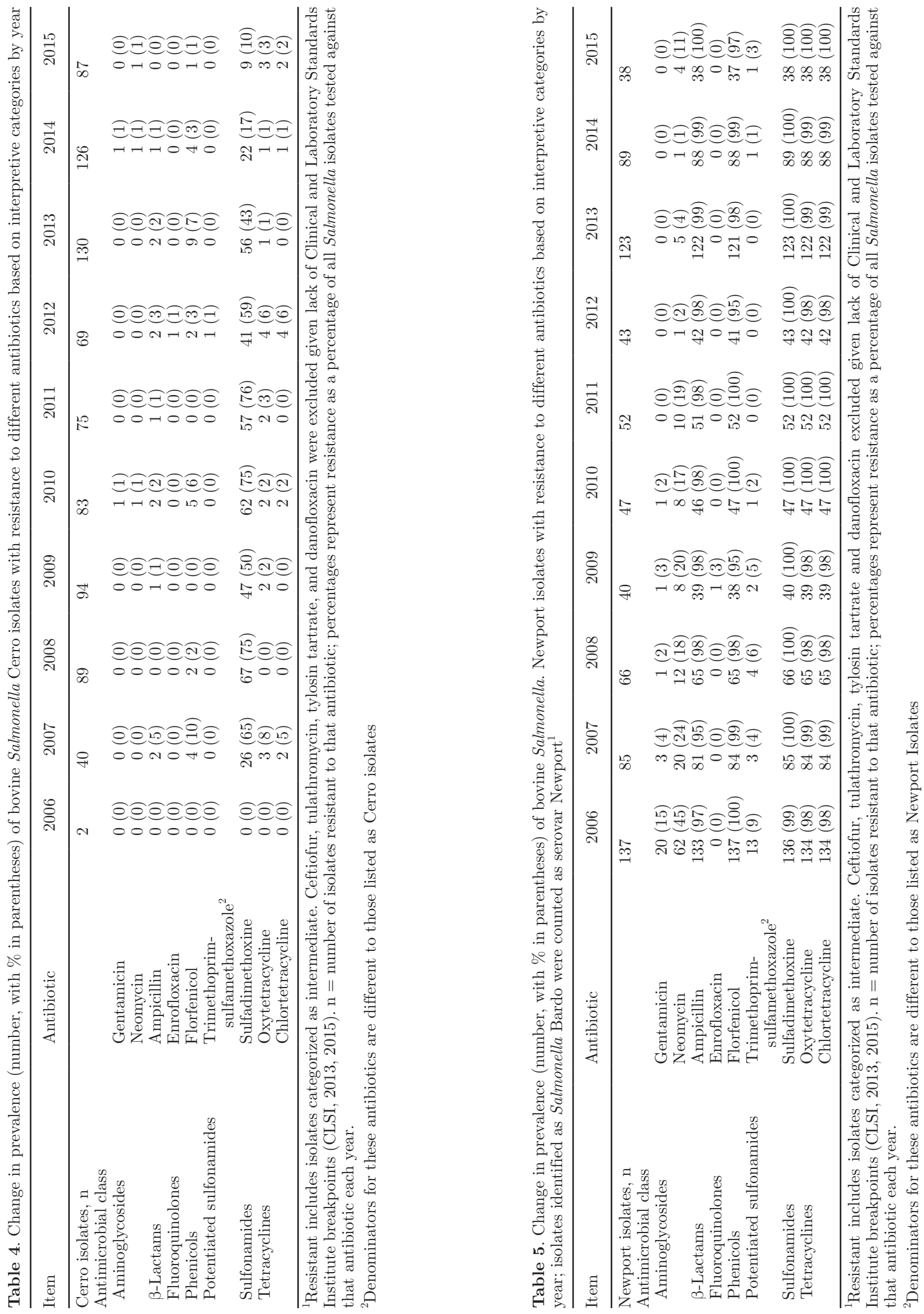
Table 6. Yearly number and percentage (\% in parentheses) of all bovine Salmonella enterica isolates tested against ceftiofur by MIC ( $\mu \mathrm{g} / \mathrm{mL})$ values

\begin{tabular}{lcccccccccc}
\hline MIC & 2006 & 2007 & 2008 & 2009 & 2010 & 2011 & 2012 & 2013 & 2014 \\
\hline$\geq 0.25$ & 0 & 1 & 3 & 0 & 0 & 1 & $4(1)$ & 1 & 0 \\
$\leq 0.5$ & $188(25)$ & $9(1)$ & 0 & 0 & 0 & 0 & 0 & 0 & 0 \\
0.5 & 0 & $116(15)$ & $146(20)$ & $126(31)$ & $123(29)$ & $87(27)$ & $83(23)$ & $104(19)$ & $104(24)$ & $88(38)$ \\
1 & $218(29)$ & $307(39)$ & $252(35)$ & $129(31)$ & $96(23)$ & $69(21)$ & $114(32)$ & $215(40)$ & $158(36)$ & $76(33)$ \\
2 & 2 & 1 & $5(1)$ & 1 & 0 & 1 & $4(1)$ & $7(1)$ & 2 & 0 \\
4 & 3 & 0 & 3 & 0 & $4(1)$ & 0 & $2(1)$ & 1 & 2 \\
8 & $62(8)$ & $74(9)$ & $41(6)$ & $17(4)$ & $27(6)$ & $18(6)$ & $16(4)$ & $13(2)$ & $19(4)$ & $9(4)$ \\
$>8$ & $287(38)$ & $275(35)$ & $264(37)$ & $140(34)$ & $174(41)$ & $145(45)$ & $135(38)$ & $194(36)$ & $150(34)$ & $58(25)$ \\
Total & 760 & 783 & 714 & 413 & 424 & 321 & 358 & 535 & 435 \\
\hline
\end{tabular}

${ }^{1}$ Percentages represent resistance as a percentage of all Salmonella isolates tested against ceftiofur each year. Assume less than $1 \%$ resistance where no percentage value listed. Kruskal-Wallis test showed that overall, changes were statistically significant $\left[\chi^{2}(9)=42.084, P=0.0001\right]$

isolates at its peak in 2007 to a 10-yr low in 2011, with only 321 isolates that were fully serotyped and for which complete AST data are available.

\section{DISCUSSION}

All of the most commonly isolated serotypes in our study were among the 10 most common serotypes of Salmonella isolated from cattle in the 2011 NARMS Annual Animal Report (USDA, 2014). A 2012 study looking at resistance in Salmonella in dairy cattle in Wisconsin also included these 6 serotypes in their most commonly detected serotypes with a sample size of 45 isolates (Marrero-Ortiz et al., 2012).

Our study found a significant decrease in resistance seen in aminoglycosides. This could be influenced by the long-standing support of a voluntary ban of the use of aminoglycoside antibiotics in cattle by several organizations, including the American Veterinary Medical Association and the American Association of Bovine Practitioners.

Susceptibility of all isolates to enrofloxacin remained high throughout our study period. Fluoroquinolones and certain sulfonamides are among those drug classes prohibited for extra-label use in food-producing animals. These restrictions may be critical to minimize development of resistance to fluoroquinolone drugs, wherein resistance has traditionally been attributed to chromosomal mutations, which are rarer as compared with transfer of genes via plasmids (Ray et al., 2007). Whereas chromosomal mutations remain the primary mechanism for resistance to these drugs in animals, a growing body of evidence demonstrates that plasmidmediated quinolone resistance is occurring (Jones-Dias et al., 2013).

Similar trends in antimicrobial susceptibility were reported in studies looking at resistance of Salmonella in dairy cattle in the United States over similar study periods (Cummings et al., 2013; Habing et al., 2015). Recently, research performed at the Wisconsin State Laboratory of Hygiene demonstrated similar findings comparing resistance levels in human salmonellosis cases from Wisconsin to national data (S. Keepman, University of Wisconsin School of Medicine and Public Health, Madison, personal communication).

The predominance of Salmonella Dublin in this population over the study period, as well as its apparent decline in recent years, is notable. Serotype Dublin is highly host-adapted to cattle and infrequently associated with human infection, but zoonosis is associated with high mortality (Mandal and Brennand, 1988). Furthermore, many Dublin isolates from human infec-

Table 7. Yearly number and percentage (\% in parentheses) of all bovine Salmonella enterica isolates tested against tulathromycin by MIC $(\mu \mathrm{g} / \mathrm{mL})$ values

\begin{tabular}{|c|c|c|c|c|c|c|c|c|c|}
\hline MIC & 2007 & 2008 & 2009 & 2010 & 2011 & 2012 & 2013 & 2014 & 2015 \\
\hline 2 & 1 & 0 & 0 & 0 & 0 & $2(1)$ & 0 & 0 & 1 \\
\hline 4 & $9(1)$ & 79 (11) & $30(7)$ & $5(1)$ & $22(7)$ & $45(13)$ & $122(23)$ & $3(1)$ & $128(56)$ \\
\hline 16 & $240(34)$ & $184(26)$ & $126(31)$ & $180(43)$ & $104(33)$ & $108(30)$ & $90(17)$ & $111(26)$ & $13(6)$ \\
\hline 32 & $13(2)$ & $5(1)$ & $20(5)$ & $57(14)$ & $15(5)$ & $9(3)$ & $7(1)$ & $40(9)$ & 1 \\
\hline 64 & 0 & 0 & 2 & 2 & 1 & 0 & 0 & 1 & 0 \\
\hline
\end{tabular}

${ }^{1}$ No data available for 2006 before tulathromycin available on susceptibility plate. Percentages represent resistance as a percentage of all Salmonella isolates tested against tulathromycin each year. Assume less than 1\% resistance where no percentage value listed. Kruskal-Wallis test showed that overall, changes were statistically significant $\left[\chi^{2}(8)=552.879, P=0.0001\right]$. 


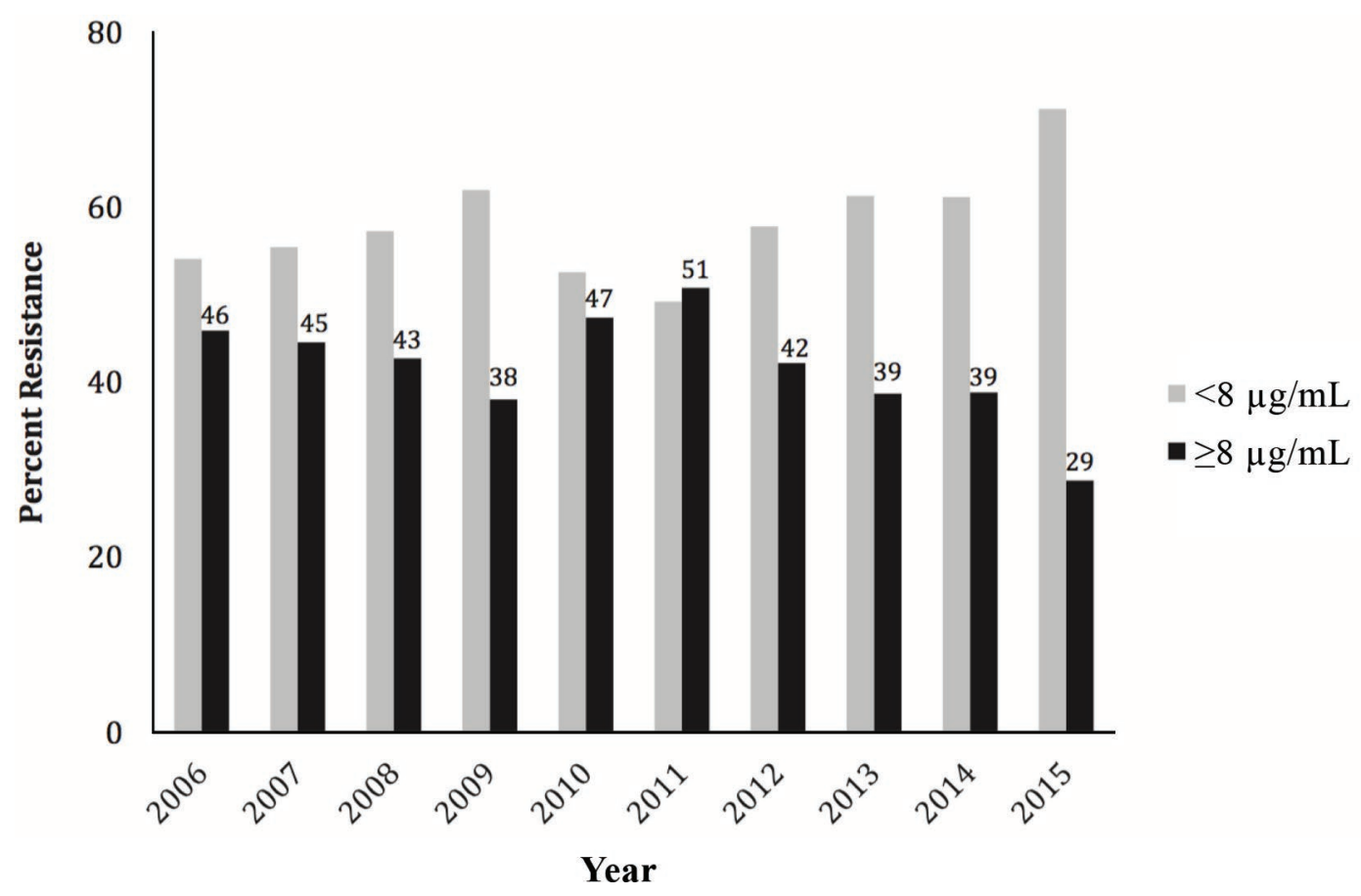

Figure 1. Change in percent resistance based on distribution of MIC values dichotomized at MIC $\geq 8 \mu \mathrm{g} / \mathrm{mL}$ for ceftiofur by year. Given the lack of Clinical and Laboratory Standards Institute (CLSI, 2013, 2015) interpretive criteria for bovine Salmonella enterica and ceftiofur, this antimicrobial agent had to be evaluated using only MIC values. National Antimicrobial Resistance Monitoring System (USDA, 2014) has reported a breakpoint for ceftiofur and Salmonella where an MIC $\geq 8 \mu \mathrm{g} / \mathrm{mL}$ is considered resistant. Full range of concentrations include $\leq 0.25$, $\leq 0.5,0.5,1,2,4,8$, and $>8$.

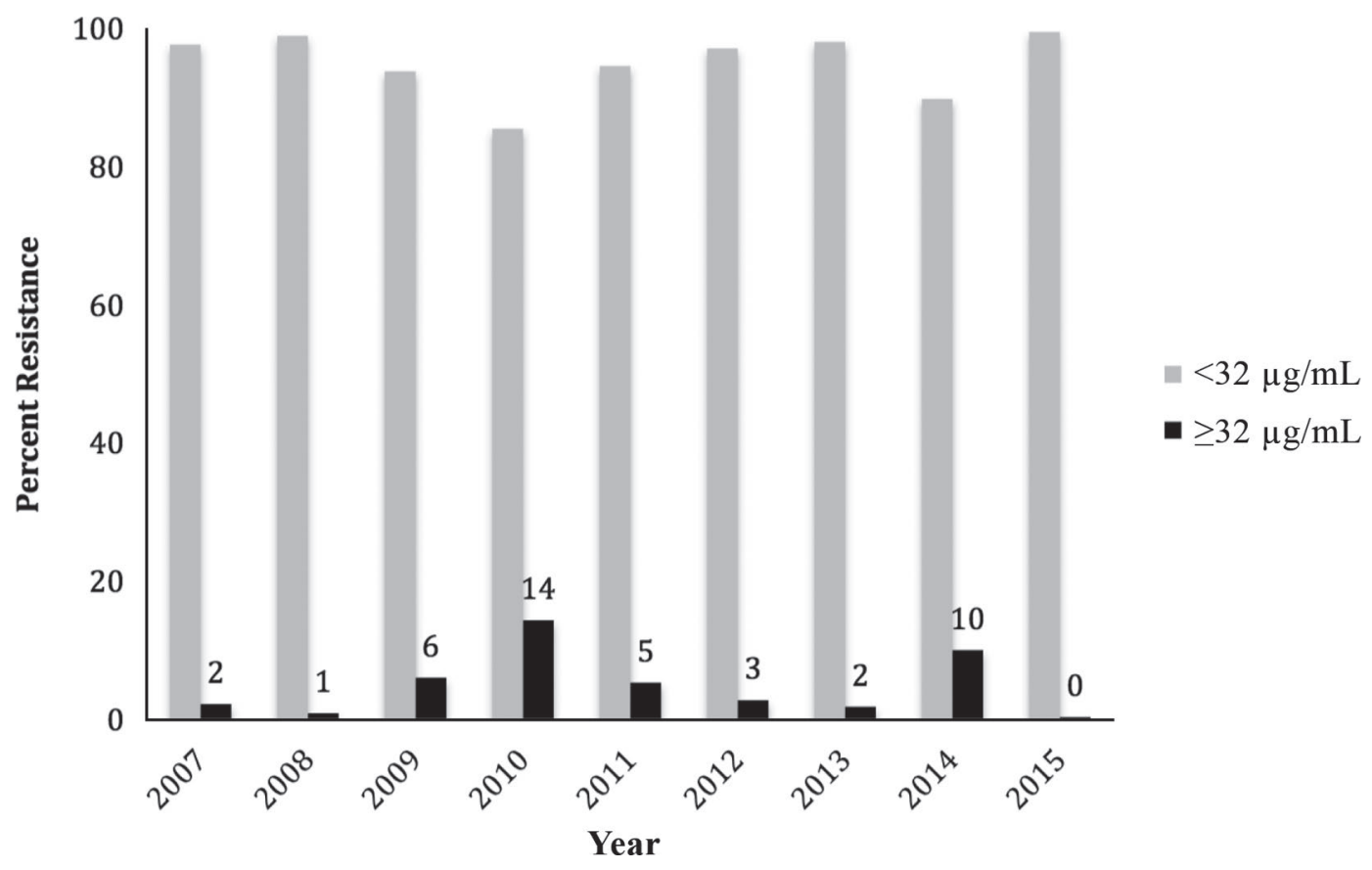

Figure 2. Change in percent resistance based on distribution of MIC values dichotomized at MIC $\geq 32 \mu \mathrm{g} / \mathrm{mL}$ for tulathromycin by year. Given the lack of Clinical and Laboratory Standards Institute (CLSI, 2013, 2015) interpretive criteria for bovine Salmonella enterica and tulathromycin, this antimicrobial agent had to be evaluated using only MIC values. National Antimicrobial Resistance Monitoring System (NARMS; USDA, 2014) has no breakpoint for tulathromycin; therefore, the NARMS breakpoint for azithromycin and Salmonella was used, whereby an MIC $>32 \mu \mathrm{g} / \mathrm{mL}$ is considered resistant. Full range of concentrations include 1, 2, 4, 8, 16, 32, 64, and $>64$. 
tions are known to be multidrug resistant (Davis et al., 2007; Lynne et al., 2009; Han et al., 2012).

We observed a reduction in the number of isolates that were identified as Dublin with complete AST results (Table 1). This could reflect a true decrease in the prevalence of Dublin, as many dairy herds in Wisconsin are moving from epidemic to endemic stages of infection. Other factors that could be influencing this trend include the adaptation of molecular diagnostics testing for Dublin using RT-PCR for identification, which is more sensitive than culture. Salmonella Dublin is difficult to isolate from fecal samples due to low shedding during infection and the fastidious nature of growth in in vitro settings with an overall culture rate of 0 to $25 \%$ (Nielsen, 2013). Whereas RT-PCR may improve detection of this organism, positive samples with high $\mathrm{C}_{\mathrm{T}}$ values, typically do not yield growth in culture and are not available for serotyping or subjected to AST. Among isolates with low $\mathrm{C}_{\mathrm{T}}$ values $(<35)$, approximately 70 to $75 \%$ were cultured post-PCR for both the Salmonella species and Salmonella Dublin multiplex PCR. Adaptation of molecular testing methodology, as related to detection of Dublin, was introduced at the diagnostic laboratory in mid-2014 to increase sensitivity and cost efficiency, but may have contributed marginally to the decreased prevalence with selective culture of suspect cases. Another potential factor in decreased prevalence could be an increased awareness of the clinical presentation, gross pathology, or historical prevalence on a particular farm of Dublin, resulting in presumptive on-farm diagnosis by the herd veterinarian or manager without confirmation through sample submission.

Our data suggest that Salmonella Cerro is an emerging serotype in this population. Cerro has increased from less than $1 \%$ of total isolates identified in 2006 to $29 \%$ of all isolates in 2014, surpassing Dublin and Newport. We also observed similar trends through July 2015. Other studies have reported increased occurrence of Salmonella Cerro in cattle regionally and nationally in recent years (Ray et al., 2007; Tewari et al., 2012; Cummings et al., 2013). Data on Salmonella Cerro from some of these studies suggest it is often associated with subclinical infections. In the 2011 NARMS Animal Arm Report (USDA, 2014), Salmonella Cerro isolates in cattle showed less than $10 \%$ resistance against those antibiotics tested. This is consistent with our findings, where isolates of Salmonella Cerro showed lower levels of resistance overall, and in particular when compared with Dublin and Newport (Tables 3, 4, and 5). In a 2014 study involving genomic analysis of 27 Cerro isolates from human cases and domesticated and wild animals in New York, Washington, and Florida, several genes normally associated with virulence in other se- rotypes were absent in Cerro (Rodriguez-Rivera et al., 2014). Those authors attributed this serotype's recent emergence to a deficiency in control measures aimed specifically at infections with Cerro, given its often subclinical presentation, rather than increased hostadaptation or antimicrobial resistance. Notably, Cerro is rarely associated with cases of food-borne illness in people (CDC, 1985, 2014).

Although it is possible that the recent emergence of Cerro may be due to natural cycling of serotypes, it is worth considering whether vaccination against certain Salmonella serotypes (i.e., Dublin specific) may be creating selection pressure, which in turn is favoring the rise of Cerro. This has previously been reported in humans with Salmonella Typhi vaccination leading to emergence of Salmonella Paratyphi (Teh et al., 2014). Other biologic products aimed at reducing disease from salmonellosis in cattle, such as gram-negative core and siderophore vaccines, could also have a selection pressure effect. Whereas these Salmonella vaccines have been used to help reduce transmission and mortality associated with salmonellosis in cattle, they are generally regarded as having little to no efficacy at reducing the prevalence of Salmonella at the farm level and providing only partial or waning protection (McGuirk and Peek, 2003; Lu et al., 2014; Cernicchiaro et al., 2016). Some Salmonella vaccines in cattle are also associated with transient negative effects on milk production (Bergeron and Elsener, 2008). Further study into the dynamics of serotype cycling and effects of vaccination on the epidemiology of Salmonella enterica are needed.

Salmonella Newport and Typhimurium are important serotypes, with respect to public health, and of particular zoonotic concern, given their incidence in the human population. Of the top 6 serotypes found in our study population, only Typhimurium, Newport, and Montevideo were among the 10 most common serotypes of non-typhoidal Salmonella in humans in 2011 (FDA, 2013; CDC, 2015). Based on data provided by Rachel Klos at the Bureau of Communicable Diseases (Wisconsin Department of Health Services, Madison, WI, personal communication), in 2013, of 904 confirmed human cases of Salmonella enterica in Wisconsin, Typhimurium and Newport were among the top 15 serotypes isolated (24 and $8 \%$ of total isolates, respectively). Salmonella Montevideo also was isolated from human salmonellosis cases in Wisconsin that year, but it accounted for less than $2 \%$ of total isolates (Rachel Klos, Wisconsin Department of Health Services, Madison, WI, personal communication).

We noted a statistically significant change overall in the MIC distribution for ceftiofur over the study period, as well as a decrease in the proportion of isolates considered resistant to ceftiofur particularly since 2012 
when using NARMS breakpoints. Ceftiofur is a thirdgeneration cephalosporin, a class of drugs important in treating severe Salmonella enterica infections in children, making any change in resistance an issue of key public health importance. Since 2012, any extralabel use of this drug in production animals is strictly prohibited. Importantly, we found this restriction coincided with the decrease in resistance observed in our study population, suggesting that regulating the use of this antibiotic may have a positive effect.

Whereas tulathromycin is not used specifically to treat salmonellosis in cattle, concern exists its widespread use for the treatment of bovine respiratory disease could be driving development of resistance and could translate to resistance to macrolides overall. In general, Salmonella species are considered to have lower susceptibility to macrolides (Pyörälä et al., 2014); resistance to macrolides in cattle, though studied primarily in organisms associated with bovine respiratory disease (e.g., Pasteurella multocida and Mannheimia haemolytica), has tended to be rare in the European Union, although it may be increasing in the United States and Canada (Pyörälä et al., 2014). Given the relatively recent adoption of this antibiotic and critical importance of this drug class in human medicine, judicious use and continued surveillance for future changes in resistance is warranted. Whereas no equivalent breakpoints have been established by NARMS for tulathromycin and Salmonella, the breakpoint used in our study for a different macrolide drug (azithromycin) results in a more conservative estimation of resistance than had the NARMS breakpoint for tulathromycin in the treatment of bovine respiratory disease ( $\mathrm{MIC} \geq 64 \mu \mathrm{g} / \mathrm{mL}$ ) been used.

There are important limitations to our study. First, changes in testing methods and susceptibility testing standards during the study period may have affected our results. The change in MIC plate format used at the diagnostic laboratories starting in 2007 could have influenced perceived changes in the proportions of isolates considered resistant resulting from changes in how those samples were tested, rather than due to true changes in the study population. Analysis was limited to only those antibiotics common to both plates. The plate change was made early on in the study period and is unlikely to have had a significant effect throughout a 10-yr study period. No obvious policy or protocol change at the diagnostic laboratory was identified to explain the trends identified in analyzing MIC distributions for antimicrobials with interpretive criteria. The questions raised by differences we found in the first 5 yr of the 10-yr study period compared with the last 5 yr warrants future study.
Additionally, as discussed, availability of RT-PCR testing for Salmonella Dublin began in 2014, which may have affected the number of isolates specific to that serotype available for susceptibility testing. Important to note is that Cerro overtook Dublin as the most prevalent serotype starting in 2013, before this change in testing methods occurred. Another limitation is related to the diagnostic laboratory's practice of grouping AST data, whereby 1 accession number may have more than 1 animal associated with it. Veterinarians often choose to submit samples from multiple animals under 1 accession to maximize the cost-to-benefit ratio and accuracy of testing on a particular farm. As a result, many different Salmonella colonies may be isolated for a given accession from more than 1 tissue or more than 1 animal. To reduce the client cost associated with a given accession, the laboratory only serotypes and performs AST on 1 of the Salmonella isolates from an accession and then serogroups, based on the O-antigen, the other Salmonella isolates to confirm the serogroup matches the fully serotyped isolate. Therefore, the quantities of Salmonella isolated and subjected to susceptibility testing are significantly reduced in this data, as isolates that were serogrouped only were not counted. This may lead to an inaccurate estimation of resistance in the population due to not all Salmonella isolates being included.

Another limitation of our study is the lack of CLSI interpretive criteria for certain antibiotics (ceftiofur, tulathromycin, danofloxacin, and tylosin tartrate), which limited analysis to numerical MIC values. Based on CLSI standards, these numbers are not interpretable, thus not allowing for conclusions to be made about an isolate's resistance. We adjusted to this limitation by using breakpoints established by NARMS (USDA, 2014) for ceftiofur and azithromycin in our analysis for ceftiofur and tulathromycin, respectively. Unfortunately, this is reflective of the lack of standardization of AST monitoring practices across human and veterinary medicine. Related to this issue is the fact that for those antibiotics with CLSI interpretive criteria breakpoints, often those breakpoints are extrapolated from human standards in the case of Salmonella enterica infections, as bovine-specific data lack for that organism.

Though the numbers of yearly isolates appear to be recovering in 2013 (535) and 2014 (435), they remain well below those at the beginning of the study period. One possible explanation for the overall drop in total isolate numbers is the vulnerability of veterinary clinical samples, particularly of production animals, to economic factors. Sample submission is dependent not only on the cost of testing, but also the ability to ship to the laboratory, milk price, and emerging trends. Given the 
dynamic nature of our study population, the influence of economic factors could result in samples submitted from larger operations being overrepresented, particularly during economically difficult years.

No information regarding herd size was available in the data we analyzed, although larger herd size has been consistently associated with a greater likelihood of identification of Salmonella on dairy farms (Fossler et al., 2005; Habing et al., 2012). Other factors, including presence of wildlife, housing, and various management practices, have also been linked to the presence of $\mathrm{Sal}$ monella (Fossler et al., 2005). In a study evaluating farm-level associations with both presence of Salmonella and antimicrobial resistant strains, Habing et al. (2012) found that specific practices related to manure management have a significant effect on the presence of Salmonella and in selecting for resistant populations, even more so than antimicrobial use. These findings are also consistent with research finding little difference in resistance levels between conventional and organic farms (Ray et al., 2006). Annual bovine Salmonella isolate totals may also be affected by the fact that samples are more likely to be submitted in epidemic phases of infection on a farm. As a disease becomes endemic, practitioners tend to recognize the disease grossly and are less likely to submit samples. Furthermore, salmonellosis in animals is not a reportable disease, which can hamper data collection from private laboratories.

\section{CONCLUSIONS}

Salmonella enterica is a major pathogen of animals and humans, with significant implications for food safety and production, veterinary medicine, and public health. The dynamic nature of Salmonella epidemiology at the local, national, and global level, including antimicrobial susceptibility, requires ongoing surveillance. Resistance was stable or decreasing between 2006 and 2015, and many factors likely influenced these trends. Some are manageable through mandatory and voluntary regulation of antibiotic use and surveillance methods, but other factors are outside regulatory control, including natural cycling of serotypes and the bacteria's adaptive resistance elements. Informed antimicrobial stewardship will require an interdisciplinary approach with continued surveillance and study. Future directions for this data include study of the trends among different subsets of the data, including comparing resistance patterns among different age and production status of dairy and beef breeds, ill and healthy animals, and different specimen types. Closer evaluation of MIC values for all antibiotics may be useful in evaluating for more subtle trends in antimicrobial resistance not observed using the CLSI interpretations. This data can also be linked to geospatial data analysis and evaluation for the presence of multidrug resistance patterns.

\section{ACKNOWLEDGMENTS}

We thank Erik Twaroski and Da Bin Lee (both from Wisconsin Veterinary Diagnostic Laboratory) for their assistance with data collection.

\section{REFERENCES}

Bergeron, R., and J. Elsener. 2008. Comparison of postvaccinal milk drop in dairy cattle vaccinated with one of two different commercial vaccines. Vet. Ther. 9:141-146.

CDC. 1985. Epidemiologic Notes and Reports Salmonellosis Associated with Carne Seca-New Mexico. Morbidity Mortality Weekly Report (MMWR). Accessed Nov. 20, 2015. http://www.cdc.gov/ mmwr/preview/mmwrhtml/00000628.htm.

CDC. 2013a. Revised Recommendations for the Interpretation of Salmonella serovar. Effective date 7/25/2013. Centers for Disease Control and Prevention, Atlanta, GA.

CDC. 2013b. Antibiotic Resistance Threats in the United States, 2013 Accessed Oct. 30, 2015. http://www.cdc.gov/drugresistance/ threat-report-2013/pdf/ar-threats-2013-508.pdf\#page=71http:// www.cdc.gov/drugresistance/threat-report-2013/pdf/arthreats-2013-508.pdf

CDC. 2014. Multiple-Serotype Salmonella Outbreaks in Two State Prisons-Arkansas, August 2012. Morbidity Mortality Weekly Report (MMWR). Accessed Nov. 20, 2015. http://www.cdc.gov/ mmwr/preview/mmwrhtml/mm6308a2.htm.

CDC. 2015. National Antimicrobial Resistance Monitoring System for Enteric Bacteria (NARMS): Human Isolates Final Report, 2013. Atlanta, Georgia. Accessed Nov. 23, 2015. http://www.cdc.gov/ narms/pdf/2013-annual-report-narms-508c.pdf

Cernicchiaro, N., S. E. Ives, T. S. Edrington, T. G. Nagaraja, and D. G. Renter. 2016. Efficacy of a Salmonella Siderophore receptor protein vaccine on fecal shedding and lymph node carriage of salmonella in commercial feedlot cattle. Foodborne Pathog. Dis. 13:517-525. https://doi.org/10.1089/fpd.2016.2129.

CLSI. 2013. Performance Standards for Antimicrobial Disk and Dilution Susceptibility Tests for Bacteria Isolated From Animals; Approved Standard. 4th ed. CLSI document VET01-A4. Clinical and Laboratory Standards Institute, Wayne, PA.

CLSI. 2015. Performance Standards for Antimicrobial Disk and Dilution Susceptibility Tests for Bacteria Isolated From Animals. 3rd ed. CLSI supplement VET01S. Clinical and Laboratory Standards Institute, Wayne, PA.

Costa, L. F., T. A. Paixão, R. M. Tsolis, A. J. Bäumler, and R. L. Santos. 2012. Salmonellosis in cattle: Advantages of being an experimental model. Res. Vet. Sci. 93:1-6. https://doi.org/10.1016/j. rvsc.2012.03.002.

Cummings, K. J., G. A. Perkins, S. M. Khatibzadeh, L. D. Warnick, and C. Altier. 2013. Antimicrobial resistance trends Among Salmonella isolates obtained from dairy cattle in the northeastern United States, 2004-2011. Foodborne Pathog. Dis. 10:353-361. https://doi.org/10.1089/fpd.2012.1285.

Davis, M. A., D. D. Hancock, T. E. Besser, J. B. Daniels, K. N K. Baker, and D. R. Call. 2007. Antimicrobial resistance in Salmonella enterica serovar Dublin isolates from beef and dairy sources. Vet. Microbiol. 119:221-230. /https://doi.org/10.1016/j. vetmic.2006.08.028.

FDA. 2013. National Antimicrobial Resistance Monitoring System for Enteric Bacteria (NARMS): 2011 Executive Report. Rockville, MD. Accessed Nov. 21, 2015. http://www.fda.gov/downloads/ AnimalVeterinary/SafetyHealth/AntimicrobialResistance/ 
NationalAntimicrobialResistanceMonitoringSystem/UCM407962. pdf.

Foley, SL, and AM Lynne.. 2007. Food animal-associated Salmonella challenges: Pathogenicity and antimicrobial resistance. J. Anim. Sci. 86:E173-E187. https://doi.org/10.2527/jas.2007-0447.

Fossler, C. P., S. J. Wells, J. B. Kaneene, P. L. Ruegg, L. D. Warnick, J. B. Bender, L. E. Eberly, S. M. Godden, and L. W. Halbert. 2005. Herd-level factors associated with isolation of Salmonella in a multi-state study of conventional and organic dairy farms. Prev. Vet. Med. 70:257-277. https://doi.org/10.1016/j. prevetmed.2005.04.003.

Haack, S. K., J. W. Duris, D. W. Kolpin, M. J. Focazio, M. T. Meyer, H. E. Johnson, R. J. Oster, and W. T. Foreman. 2016. Contamination with bacterial zoonotic pathogen genes in U.S. streams influenced by varying types of animal agriculture. Sci. Total Environ. 563-564:340-350. https://doi.org/10.1016/j.scitotenv.2016.04.087 (Epub.).

Habing, G. G., J. E. Lombard, C. A. Kopral, D. A. Dargatz, and J. B. Kaneene. 2012. Farm-level associations with the shedding of Salmonella and antimicrobial-resistant Salmonella in U.S. dairy cattle. Foodborne Pathog. Dis. 9:815-821. https://doi.org/10.1089/ fpd.2012.1149.

Habing, G. G., S. Manning, C. Bolin, Y. Cui, J. Rudrik, S. Dietrich, and J. B. Kaneene. 2015. Within-farm changes in dairy farm-associated Salmonella subtypes and comparison to human clinical isolates in Michigan, 2000-2001 and 2009. Appl. Environ. Microbiol. 81:5724-5735. https://doi.org/10.1128/AEM.00899-15

Han, J., A. M. Lynne, D. E. David, R. Nayak, and S. L. Foley. 2012. Sequencing of plasmids from a multi-antimicrobial resistant Salmonella enterica serovar Dublin strain. Food Res. Int. 45:931-934. https://doi.org/10.1016/j.foodres.2011.04.016.

Huston, C. L., T. E. Wittum, B. C. Love, and J. E. Keen. 2002. Prevalence of fecal shedding of Salmonella spp in dairy herds. J. Am. Vet. Med. Assoc. 220:645-649.

Jay-Russell, MT, AF Hake, Y Bengson, A Thiptara, and T Nguyen.. 2014. Prevalence and characterization of Escherichia coli and Salmonella strains isolated from stray dog and coyote feces in a major leafy greens production region at the United States-Mexico border. PLoS One 9:e113433. https://doi.org/10.1371/journal. pone. 0113433 .

Jones-Dias, D., V. Manageiro, A. P. Francisco, A. P. Martins, G. Domingues, D. Louro, E. Ferreira, and M. Caniça. 2013. Assessing the molecular basis of transferable quinolone resistance in Escherichia coli and Salmonella spp. from food-producing animals and food products. Vet. Microbiol. 167:523-531. https://doi. org/10.1016/j.vetmic.2013.08.010.

Lu, Z., Y. T. Gröhn, R. L. Smith, J. S. Karns, E. Hovingh, and Y. H. Schukken. 2014. Stochastic modeling of Imperfect Salmonella vaccines in an adult dairy herd. Bull. Math. Biol. 76:541-565. https:// doi.org/10.1007/s11538-013-9931-5.

Lynne, A. M., L. L. Dorsey, D. E. David, and S. L. Foley. 2009. Characterisation of antibiotic resistance in host-adapted Salmonella enterica. Int. J. Antimicrob. Agents 34:169-172. https://doi. org/10.1016/j.ijantimicag.2009.02.018.

Mandal, B. K., and J. Brennand. 1988. Bacteraemia in salmonellosis: A 15 year retrospective study from a regional infectious diseases unit. BMJ 297:1242-1243.

Marrero-Ortiz, R., J. Han, A. M. Lynne, D. E. David, M. E. Stemper, D. Farmer, W. Burkhardt, R. Nayak, and S. L. Foley. 2012. Genetic characterization of antimicrobial resistance in Salmonella Enterica serovars isolated from dairy cattle in Wisconsin. Food Res. Int. 45:962-967. https://doi.org/10.1016/j.foodres.2011.04.013.

McGuirk, S. M., and S. Peek. 2003. Salmonellosis in cattle: A review. In 36th Annual Conference of the American Association of Bovine
Practitioners, Columbus, OH. Accessed Dec. 21, 2015. http:// www.vetmed.wisc.edu/dms/fapm/fapmtools/7health/Salmorev. pdf.

Nielsen. L. R. 2013. Review of pathogenesis and diagnostic methods of immediate relevance for epidemiology and control of Salmonella Dublin in cattle. Vet. Microbiol. 162:1-9. https://doi. org/10.1016/j.vetmic.2012.08.003.

Nielsen, T. D., L. E. Green, A. B. Kudahl, S. Østergaard, and L. R. Nielsen. 2012. Evaluation of milk yield losses associated with Salmonella antibodies in bulk tank milk in bovine dairy herds. J. Dairy Sci. 95:4873-4885. https://doi.org/10.3168/jds.2011-4332.

Nielsen, T. D., A. B. Kudahl, S. Østergaard, and L. R. Nielsen. 2013 Gross margin losses due to Salmonella Dublin infection in Danish dairy cattle herds estimated by simulation modeling. Prev. Vet. Med. 111:51-62. https://doi.org/10.1016/j.prevetmed.2013.03.011.

O'Doherty, E., R. Sayers, L. O' Grady, and L. Shalloo. 2015. Effect of exposure to Neospora caninum, Salmonella, and Leptospira interrogans serovar Hardjo on the economic performance of Irish dairy herds. J. Dairy Sci. 98:2789-2800. https://doi.org/10.3168/ jds.2014-8168.

Peters, A. R. 1985. An estimation of the economic impact of an outbreak of Salmonella Dublin in a calf rearing unit. Vet. Rec. 117:667-668.

Pyörälä, S., K. E. Baptiste, B. Catry, E. van Duijkere, C. Greko, M. A Moreno, M. C. Pomba, M. Rantala, M. Ružauskas, P. Sanders, E. J. Threlfall, J. Torren-Endo, and K. Törneke. 2014. Macrolides and lincosamides in cattle and pigs: Use and development of antimicrobial resistance. Vet J. 200:230-239. https://doi.org/10.1016/j. tvjl.2014.02.028.

Ray, K. A., L. D. Warnick, R. M. Mitchell, J. B. Kaneene, P. L. Ruegg, S. J. Wells, C. P. Fossler, L. W. Halbert, and K. May. 2006. Antimicrobial susceptibility of Salmonella from organic and conventional dairy farms. J. Dairy Sci. 89:2038-2050. https://doi. org/10.3168/jds.S0022-0302(06)72271-8.

Ray, K. A., L. D. Warnick, R. M. Mitchell, J. B. Kaneene, P. L. Ruegg, S. J. Wells, C. P. Fossler, L. W. Halbert, and K. May. 2007. Prevalence of antimicrobial resistance among Salmonella on Midwest and northeast USA dairy farms. Prev. Vet. Med. 79:204-223. https://doi.org/10.1016/j.prevetmed.2006.12.001.

Rodriguez-Rivera, L. D., A. I. M. Switt, L. Degoricija, R. Fang, C. A. Cummings, M. R. Furtado, M. Wiedman, and H. C. den Bakker. 2014. Genomic characterization of Salmonella Cerro ST367, an emerging Salmonella subtype in cattle in the United States. BMC Genomics 15:427. https://doi.org/10.1186/1471-2164-15-427.

Teh, C. S. J., K. H. Chua, and K. L. Thong. 2014. Paratyphoid fever: Splicing the global analyses. Int. J. Med. Sci. 11:732-741. https:// doi.org/10.7150/ijms.7768.

Tewari, D., C. H. Sandt, D. M. Miller, B. M. Jayarao, and N. M. M'ikanatha. 2012. Prevalence of Salmonella Cerro in laboratorybased submissions of cattle and comparison with human infections in Pennsylvania, 2005-2010. Foodborne Pathog. Dis. 9:928-933. https://doi.org/10.1089/fpd.2012.1142.

USDA. 2014. National Antimicrobial Resistance Monitoring System for Enteric Bacteria (NARMS): 2011 NARMS Animal Arm Annual Report. Athens, GA. Accessed Nov. 20, 2015. http://www.ars. usda.gov/SP2UserFiles/Place/60400520/NARMS/NARMS2011/ NARMS\%20USDA\%202011\%20Report.pdf

Van Kessel, J. S., J. Sonnier, S. Zhao, and J. S. Karns. 2013. Antimicrobial resistance of Salmonella Enterica isolates from bulk tank milk and milk filters in the United States. J. Food Prot. 76:18-25. https://doi.org/10.4315/0362-028X.JFP-12-263. 